\title{
Breast Cancer: Knowing is More Important than Wondering
}

\section{Richard M Fleming ${ }^{1 *}$, Matthew R Fleming ${ }^{1}$, William C Dooley ${ }^{2}$ and Tapan K Chaudhuri ${ }^{3}$}

${ }^{1}$ FHHI-Omnific Imaging-Camelot, El Segundo, CA, USA

${ }^{2}$ Oklahoma University Health Science Center, Oklahoma City, OK, USA

${ }^{3}$ Eastern Virginia Medical School, Norfolk, VA, USA

*Corresponding Author: Richard M Fleming, FHHI-Omnific Imaging-Camelot, El Segundo, CA, USA.

Received: September 24, 2019; Published: October 05, 2019

DOI: $10.31080 /$ ASMS.2019.03.0431

In a recent USPSTF statement [1], the task force recommended that women with a familial and potential genetic predisposition for BRCA1/2 gene mutations be tested and undergo genetic counseling.

Multiple cancers, like many hyperlipidemias, are associated with genetic predispositions, including BRCA1/2, p53 and Lynch Syndrome [2]. However, these genetic predispositions toward transitional tissue changes do not mean cancer is destined to develop [3]. How do you counsel someone over what might happen, without knowing which environmental factors will set into motion the chain of events for that particular individual, which will produce cancer [3]?

Henry Lynch was someone the primary author knew and worked with. Henry was more concerned with how genetic tests like BRCA1/2 would be used. At best, they demonstrate the potential for cancer and at worse it causes women and men to be fearful of dying from a cancer, which they may not, at least as of the time of genetic testing, have. As we know, women (and men) with BRCA1/2 account for only 5 to $10 \%$ of all breast cancers [4].

Today one of the driving concerns in medicine is the failure to find breast cancer when present (sensitivity errors) and the inability to guarantee that there is no cancer present (specificity) when none is seen. This limitation and consequential patient (and family) fear, is driven by qualitative tests such as mammography, which has proven to be unreliable in half of all women, specifically including the $50 \%$ of women who have dense breasts.

The task force statement brings to light some of the potential problems associated with genetic testing, followed by the limitations of qualitative imaging. Do you or don't you have cancer?

As we have seen, this presupposes a certain mode of thinking. Either the person is at one extreme on a Health-Spectrum [3], or the other, without acknowledging transitional changes; changes which are potentially treatable. To clearly counsel patients and their families, it is important that we provide them with this information [5].
Henry was about knowing and not guessing. The BRCA1/2 test was designed to raise awareness of potential problems, but what counseling do you provide to someone who may develop cancer? Our answer is determined by our ability to measure these transitional changes as they are happening [5]. Only then can we provide the necessary counseling patients and their families' need.

\section{Conflict of Interest}

FMTVDM patent was issued to first author.

\section{Bibliography}

1. US Preventive Services Task Force. "Risk Assessment, Genetic Counseling, and Genetic Testing for BRCA-Related Cancer. US Preventive Services Task Force Recommendation Statement". Journal of the American Medical Association 322.7 (2019): 652-665.

2. American Cancer Society (ACS). Cancer Facts and Figures (2018).

3. Fleming RM., et al. "Cancer: Our Body's Global Warming Warning. Biomed Research". Open Access Journal of Oncology and Medicine 3.1 (2019): 238-239.

4. Anglian Breast Cancer Study Group. "Prevalence and penetrance of BRCA1 and BRCA2 mutations in a population-based series of breast cancer cases". British Journal of Cancer 83.10 (2000): 1301-1308.

5. Fleming RM., et al. "FMTVDM-BEST@® Breast Cancer Imaging eliminates the fear of having BRCA1 and BRCA2 Breast Cancer Genes". Journal of Clinical and Molecular Medicine 1.2 (2018):1-2.

Volume 3 Issue 11 November 2019

(C) All rights are reserved by Richard $M$ Fleming., et al. 